Dental Manifestations of Pediatric Bone Disorders

Juan F. Yepes ${ }^{1 *}$ jfyepes@iupui.edu

1

Department of Pediatric Dentistry

Attending Riley Hospital for Children, Indiana University School of Dentistry

Indianapolis, IN, USA

\title{
Abstract
}

\section{Purpose of Review}

Several bone disorders affecting the skeleton often are manifest in the maxillofacial region. This review presents the most common bone disorders in children and their dental-oral manifestations: fibrous dysplasia, Paget's disease, osteogenesis imperfecta, renal osteodystrophy, hypophosphatasia, and osteoporosis. The specific intraoral characteristics will reviewed in detail.

\section{Recent Findings}

Recent studies confirmed the close relationship between the mandible and the maxilla with the most prevalent systemic bone disorders in children.

\section{Summary}

This review will help practitioners to integrate the oral health into the systemic health and improve the multidisciplinary approach of pediatric patients between medicine and dentistry.

\section{Keywords}

Osteogenesis imperfect

Osteoporosis

This is the author's manuscript of the article published in final edited form as:

Yepes, J. F. (2017). Dental Manifestations of Pediatric Bone Disorders. Current Osteoporosis Reports, 15(6), 588592. https://doi.org/10.1007/s11914-017-0409-5 
Fibrous dysplasia

Paget's disease

Renal osteodystrophy

This article is part of the Topical Collection on Craniofacial Skeleton

\section{Introduction}

\section{Fibrous Dysplasia}

Fibrous dysplasia (FD) is the consequence of a proliferation and expansion of the bone marrow by fibrous tissue that contains an immature bone. Different components of bone structure are replaced by fibrous tissue containing different amounts of abnormal osseous tissue [1]. This condition is caused by activation of a mutation in the GNAS gene. The consequence of the GNAS activation is an overproduction of cAMP signaling pathway which decreases the ability of the immature bone cells to move into mature osteogenic cells [2]. Fibrous dysplasia may have solitary or multiple foci and may occur as a component of McCune-Albright syndrome. The long bones are most commonly involved, and the majority of lesions are symptomatic but the diagnosis may be an incidental finding during routine radiographic examination.

The physiopathology of FD involves the replacement of the normal bone and bone marrow with fibro-osseous lesions. From a histology perspective, lesions consist of immature skeletal progenitor cells, which continue to proliferate in their multipotent state without differentiating into osteoblasts, adipocytes, or cells of the hematopoietic-support lines. Microscopically, an FD lesion is characterized by marrow fibrosis, abnormal trabeculae, and formation of abnormal mineralized tissue matrix $[3,4]$.

The clinical presentation of FD in children varies according to the location and extension of the lesions. The proximal femur and skull base are the bones more frequently involved. Fractures and expansion are often present with FD [5]. The diagnosis of FD is based on radiographic and in some cases, histological evaluation. Technetium-99 scintigraphy is helpful for identifying the foci of skeletal involvement. A comprehensive laboratory evaluation is mandatory in cases of suspected FD. Evaluation includes screening for hypophosphatemia, hyperthyroidism, and 
abnormal growth hormone levels [6]. Treatment of FD is usually surgical [7].

Bisphosphonates are also used for pain control [6].

\section{Dental Manifestations}

Fibrous dysplasia in children (and adults) affects the maxillofacial region. The most common involved bones are the cranial base of the skull, maxilla, and midface [8]. The lesions can be extensive causing expansion of the skull, maxilla, or mandible. Teeth displacement associated with malocclusion and midface deformities are also present [6]. In some severe cases, FD is associated with hypoplasia and hypomineralization of teeth. The latter is related to the high prevalence of caries observed in some FD patients. The typical radiographic pattern is a "groundglass" radiopaque appearance, with an ill-defined and non-corticated morphology (Fig.11).

\section{Paget's Disease}

Paget's disease (PD) is a common bone disease in adults but uncommon in children. In children, PD is classified as juvenile Paget's disease. In general, PD is characterized by an alteration in the critical homeostasis of the osteoblast and osteoclast. The condition is often asymptomatic and detected during routine radiographic evaluations [9]. Recently, the prevalence of PD is decreasing worldwide [10-12]. Probably, the most common manifestation of the disease is skeletal deformities usually visible in the skull and lower extremities. Pathological fractures occur often and most likely in the femur. Pain is common in patients with PD from skeletal, muscle $_{2}$ or neurological origin [13•]. The primary lesion in PD is a localized area of increased bone resorption together with hypertrophy of an immature new bone. The consequence is bone expansion with structural weakness. The etiology of PD is multifactorial. Both genetic and environmental factors have been related with the initiation and progression of the condition. Among the environmental factors, dietary deficiencies and chronic infection with paramyxoviruses are the most relevant etiology for PD [14]. Gene mutations have been also identified with the development of PD, in particular genes mediating cytokine signaling [15]. Juvenile Paget's disease has a strong genetic predisposition.

Juvenile Paget's disease (JPD) is an autosomal recessive disorder associated with increased bone turnover due to activation of the osteoclast in the bone matrix. JPD affects all the bones and 
causes a progressive deformities, short stature, growth retardation, as well as facial deformities such as maxillary expansion and progressive macrocephaly. Because of the bone expansion, other structures are affected especially the auditory and optic nerves. The morbidity and mortality of JPD is high.,The majority of patients are in wheel chair before the age of 15 if the condition is not treated [16]. Oral bisphosphonates are the treatment of choice for JPD. Early treatment with these medications decreases the bone turnover and prevents the development of deformities [17].

\section{Osteogenesis Imperfecta}

Osteogenesis imperfecta (OI) is an uncommon genetic bone disorder associated with the fragile (brittle) bones and fractures in children and adults. Several genes have been identified as possible etiology for this condition; some of the genes are associated with the collagen production. OI occurs in approximately 1 in 10,000 to 20,000 live births [18]. The typical clinical presentation is bone fragility which may be associated with skeletal deformities, joint laxity, and scoliosis. Several classifications (subtypes) of OI have been proposed according to the phenotype and genotype of the clinical history. Based on the phenotype and mode of inheritance, OI is classified in four subtypes. The most severe form is OI type II presenting with extreme bone fragility and multiple spontaneous fractures. The majority of patients with OI type II die before 4 weeks of age [19]. The most common subtype is OI type I, which is associated mild to moderate bone fragility. The risk of fractures and fragility of osseous tissue is a consequence of a combination of decreased bone quality and reduced bone mass [20]. The main goal for the treatment of OI is to decrease the risk of fractures and pain. With children, physical therapy and rehabilitation are critical therapeutic factors for avoiding fractures and improving weight bearing tolerance and increasing bone strength [21].

\section{Dental Manifestations}

The dental manifestations of OI are similar to the presentation of dentinogenesis imperfecta. The primary and adult dentition are affected. The hue of OI teeth is blue to brown translucent (Fig. 2). The Teeth are smaller than normal, and a constriction of the cementum-enamel junction is present. Root size is decreased compared to a normal teeth. Furthermore, pulp chambers are progressively diminished and in some cases, completely obliterated [22]. Discoloration is less 
pronounced in the maxillary permanent incisors compared to the primary teeth. Also, children with OI present with an increased prevalence of class III malocclusion probably related to an underdeveloped maxilla [19] (Fig. 2),

\section{Renal Osteodystrophy}

The term renal osteodystrophy (RO) is used to identify several osseous conditions associated with chronic kidney disease (CKD). In children, CKD is the usual end point of congenital, acquired, inherited, or metabolic renal disease. The underlying etiology is related to the age of the patient. In children 5 years old or less, the most common cause of CKD is congenital renal hypoplasia, congenital nephrotic syndrome, polycystic kidney disease, obstructive uropathy, etc. After 5 years of age, acquired diseases increasingly interact with inherited disorders to produce the most common etiology of CKD.

Renal osteodystrophy in children is a high-turnover metabolic bone disease caused by secondary hyperparathyroidism related to $\mathrm{CKD}$. The pathophysiology of $\mathrm{RO}$ reflects a chronic decrease in the glomerular filtration rate (GFR). The decrease in functional kidney mass leads to a decline in renal $1 \alpha$-hydroxylase activity, with a decreased production of the active metabolite of vitamin D (1,25 dihydroxycholecalciferol). The decline in the active metabolite of vitamin D results in a decrease of the gastrointestinal absorption of calcium, which depresses ionic calcium $\left(\mathrm{Ca}^{++}\right)$in the serum triggering an increased secretion of parathyroid hormone [23]. The cascade of metabolic events includes increased production and function of osteoclasts to help increase serum $\mathrm{Ca}^{++}$levels. The clinical manifestations of RO include muscle weakness, bone pain, and relatively atraumatic fractures. Alterations in skeletal mineralization are common in children with RO, and the primary treatment for the disease is the administration of vitamin $\mathrm{D}$. The main objectives for treatment are to prevent bone deformity and normalize growth velocity [23].

\section{Dental Manifestations}

Renal osteodystrophy is associated with several clinical manifestations in the oral-facial complex, and the most common is diffuse jaw enlargement [24]. Radiographic changes in RO are similar to the changes observed in fibrous dysplasia or Paget's disease. This pathophysiology is consistent with ground-glass bone appearance, hypercementosis and bone resorption with loss 
of the lamina dura [25]. The latter is a common dental radiographic feature for all high-turnover metabolic bone diseases. Renal osteodystrophy is also associated with a delayed eruption pattern, dental spacing, tooth mobility, propensity for jaw fractures, and poor definition of anatomical landmarks [26].

\section{Hypophosphatasia}

Hypophosphatasia (HPP) is an inherited rare bone condition characterized by hypomineralization. This condition is classified into six subtypes according to age of onset: perinatal (lethal), perinatal benign, infantile, childhood, adult, and odontohypophosphatasia. Hypophosphatasia is characterized by a deficiency of tissue-nonspecific alkaline phosphatase (TNAP) [27]. This enzyme is critical for tissue mineralization. Lack of the enzyme carries several metabolic consequences including hypomineralization of bones and teeth [28]. Bone fragility and deformities are typical features of this condition. During childhood, symptoms are problems in walking, growth retardation, and muscle weakness [29].

\section{Dental Manifestations}

Premature loss of primary teeth (usually before the age of 3) is a key feature of hypophosphatasia (Fig. 3). The teeth usually exfoliate with intact roots. Early mobility of primary teeth is one of the first signs. As soon as the problem is noted the child should be examined by a dentist, preferably a pediatric dentist, particularly if the child is < 4 years of age. In older patients, hypophosphatasia is characterized by early loss of permanent teeth. Periodontal disease without substantial dental plaque has been reported for patients with hypophosphatasia [30]. Abnormal cementum is noted on teeth that are exfoliated prematurely and may be the primary factor in deterioration of the periodontium leading to early exfoliation [31]. Eating disorders associated with hypercalcemia and increased caries prevalence may be clinical features of hypophosphatasia [32] (Fig. 3),

\section{Osteoporosis}

Osteoporosis is not common in children, but there has been a significant increase in the diagnosis of the disorder in children in the last 5years [33]. In general, patients with juvenile osteoporosis suffer from a decrease in bone volume that is associated with increased risk for fractures [6]. 
Histologic analysis of bone biopsies for differing manifestations of juvenile osteoporosis reveals that osseous tissue mineralizes normally, but there is a sharp reduction in bone mass, particularly with respect to the trabecular bone.

Osteoporosis in children can be primary or secondary to chronic diseases. Primary osteoporosis occurs because of an intrinsic skeletal defect of genetic or idiopathic origin [34•]. The most common causes of secondary osteoporosis are immobility, leukemia, inflammation, hypogonadism, and poor nutrition. Bone fragility with spontaneous or pathologic fractures is the usual clinical presentation of osteoporosis in children. The diagnosis of juvenile osteoporosis includes laboratory blood work, e.g.r serum alkaline phosphatase, calcium phosphate, and vitamin D. Dual energy X-ray absorptiometry (DXA) is the gold standard in the diagnosis of osteoporosis [34•].Early intervention is fundamental to decrease the progression of the condition. Bisphosphonates are the treatment of choice for pediatric osteoporosis [35].

\section{Dental Manifestations}

Osteoporosis in children features similar maxillofacial characteristics as those noted in osteoporotic adults. Radiographic characteristics include a thin mandibular inferior cortex together with a decrease in the number and thickness of trabecular bone plates. Specific erosions into or through the inferior border of the mandible are common in children with osteoporosis [36].

\section{Conclusion}

Dental manifestation of skeletal disease in children is most commonly associated with fibrous dysplasia, juvenile Paget's disease, osteogenesis imperfecta, renal osteodystrophy, hypophosphatasia, and osteoporosis. In general, the systemic manifestations of skeletal disease in children is similar to adults, but the rapid dentofacial growth and development of children render the orofacial region particularly vulnerable to specific signs and symptoms. It is important for parents and health professionals to carefully monitor the orofacial region for unusual form and function. Dental problems may be the first significant manifestation of serious skeletal disease. 


\title{
Compliance with Ethical Standards
}

\author{
Conflict of Interest
}

Juan Yepes declares no conflict of interest.

\section{Human and Animal Rights and Informed Consent}

This article does not contain any studies with human or animal subjects performed by any of the authors.

\section{References}

\section{Papers of particular interest, published recently, have been highlighted as: • Of importance}

1. Anitha, N., et al., Fibrous dysplasia-recent concepts. J Pharm Bioallied Sci, 2015. 7(Suppl 1): p.

S171-2.

2. Weinstein, L.S., et al., Activating mutations of the stimulatory G protein in the McCune-Albright syndrome. N Engl J Med, 1991. 325(24): p. 1688-95.

3. Riminucci, M., et al., Osteoclastogenesis in fibrous dysplasia of bone: in situ and in vitro analysis of IL-6 expression. Bone, 2003. 33(3): p. 434-42.

4. Riminucci, M., et al., The histopathology of fibrous dysplasia of bone in patients with activating mutations of the Gs alpha gene: site-specific patterns and recurrent histological hallmarks. J Pathol, 1999. 187(2): p. 249-58.

5. Hart, E.S., et al., Onset, progression, and plateau of skeletal lesions in fibrous dysplasia and the relationship to functional outcome. J Bone Miner Res, 2007. 22(9): p. 1468_74.

6. Foster, B.L., et al., Rare bone diseases and their dental, oral, and craniofacial manifestations. J Dent Res, 2014. 93(7 Suppl): p. 7S-19S. 
7. Stanton, R.P., et al., The surgical management of fibrous dysplasia of bone. Orphanet J Rare Dis, 2012. 7 Suppl 1: p. S1.

8. Kelly, M.H., B. Brillante, and M.T. Collins, Pain in fibrous dysplasia of bone: age-related changes and the anatomical distribution of skeletal lesions. Osteoporos Int, 2008. 19(1): p. 57-63.

9. Lalam, R.K., V.N. Cassar-Pullicino, and N. Winn, Paget djsease of bुone. Semin Musculoskelet Radiol, 2016. 20(3): p. 287_299.

10. Cundy, H.R., et al., Paget's disease of bone in New Zealand: continued decline in disease severity. Calcif Tissue Int, 2004. 75(5): p. 358-64.

11. Tiegs, R.D., et al., Long-term trends in the incidence of Paget's disease of bone. Bone, 2000. 27(3): p. $423-7$.

12. Poor, G., et al., Epidemiology of Paget's disease in Europe: the prevalence is decreasing. J Bone Miner Res, 2006. 21(10): p. 1545-9.

13• Galson, D.L. and G.D. Roodman, Pathobiology of Paget’s djsease of bone. J Bone Metab, 2014. 21(2): p. 85-98. This study provides a detail description of the new and more recent knowledge about the physiopathology of Paget's disease.

14. Ralston, S.H. and R. Layfield, Pathogenesis of Paget disease of bone. Calcif Tissue Int, 2012. 91(2): p. $97-113$.

15. Roodman, G.D., Insights into the pathogenesis of Paget's disease. Ann N Y Acad Sci, 2010. 1192: p. $176-80$.

16. Indumathi, C.K., C. Dinakar, and R. Roshan, Juvenile Paget's disease. Indian Pediatr, 2009. 46(4): p. 354-6.

17. Wendlova, J., S. Galbavy, and J. Paukovic, Paget's disease of bone_treatment with alendronate, calcium and calcitriol. Vnitr Lek, 1999. 45(10): p. 602-5.

18. Monti, E., et al., Current and emerging treatments for the management of osteogenesis imperfecta. Ther Clin Risk Manag, 2010. 6: p. 367-81.

19. Damm, D.D., Oral pathology. Oral Surg Oral Med Oral Pathol, 1994. 77(1): p. 4-5. 
20. Shaker, J.L., et al., Recent developments in osteogenesis imperfecta. F1000Res, 2015. 4(F1000 Faculty Rev): p. 681.

21. Hoyer-Kuhn, H., et al., A specialized rehabilitation approach improves mobility in children with osteogenesis imperfecta. J Musculoskelet Neuronal Interact, 2014. 14(4): p. 445-53.

22. Majorana, A., et al., Dentinogenesis imperfecta in children with osteogenesis imperfecta: a clinical and ultrastructural study. Int J Paediatr Dent, 2010. 20(2): p. 112-8.

23. Kliegman, R. and W.E. Nelson, Nelson textbook of pediatrics. 19 ed. 2011, Philadelphia: Elsevier/Saunders. lxvii, 2610 p.

24. Kalyvas, D., et al., Localized jaw enlargement in renal osteodystrophy: report of a case and review of the literature. Oral Surg Oral Med Oral Pathol Oral Radiol Endod, 2004. 97(1): p. 68-74.

25. Lopes, M.L., et al., Severe maxillofacial renal osteodystrophy in two patients with chronic kidney disease. Oral Maxillofac Surg, 2015. 19(3): p. 321-7.

26. Antonelli, J.R. and T.L. Hottel, Oral manifestations of renal osteodystrophy: case report and review of the literature. Spec Care Dentist, 2003. 23(1): p. 28-34.

27. Orimo, H., Pathophysiology of hypophosphatasia and the potential role of asfotase alfa. Ther Clin Risk Manag, 2016. 12: p. 777_86.

28. Anderson, H.C., et al., Matrix vesicles in osteomalacic hypophosphatasia bone contain apatite-like mineral crystals. Am J Pathol, 1997. 151(6): p. 1555-61.

29. Bloch-Zupan, A., Hypophosphatasia: diagnosis and clinical signs $\Longrightarrow$ a dental surgeon perspective. Int J Paediatr Dent, 2016. 26(6): p. 426-438.

30. Schlosser, B.J., M. Pirigyi, and G.W. Mirowski, Oral manifestations of hematologic and nutritional diseases. Otolaryngol Clin N Am, 2011. 44(1): p. 183-203, vii.

31. Foster, B.L., et al., Central role of pyrophosphate in acellular cementum formation. PLoS One, 2012. 7(6): p. e38393.

32. Mornet, E., Hypophosphatasia. Orphanet J Rare Dis, 2007. 2: p. 40.

33. Marrani, E., et al., Pediatric osteoporosis: diagnosis and treatment ç considerations. Drugs, 2017. 77(6): p. 679-695. 
34•. Saraff, V. and W. Hogler, Endocrinology and adolescence: osteoporosis in children: diagnosis and management. Eur J Endocrinol, 2015. 173(6): p. R185-97. This article provides to the reader a detailed and completed review in the most updated knowledge of osteoporosis in children.

35. Russell, R.G., Bisphosphonates: mode of action and pharmacology. Pediatrics, 2007. 119 Suppl 2: p. S150-62.

36. Dervis, E., Oral implications of osteoporosis. Oral Surg Oral Med Oral Pathol Oral Radiol Endod, 2005. 100(3): p. 349-56.

Fig. 1 Fibrous dysplasia; right mandible (yellow arrows)

Fig. 2 Mandibular incisors of a 15-year-old girl affected with osteogenesis imperfecta

Fig. 3 Hypophosphatasia in a 3.5-year-old girl. Early loss of primary mandibular incisors 